\title{
Therapeutic drug monitoring in a developing country: an overview
}

\author{
N. J. Gogtay, N. A. Kshirsagar \& S. S. Dalvi \\ Department of Clinical Pharmacology, Seth Gordhandas Sunderdas Medical College \& King Edward Memorial Hospital, Parel, Mumbai, India
}

\begin{abstract}
Therapeutic Drug Monitoring (TDM) was introduced in India in the mid and late 1980s and the last 10 years have seen it grow, together with the growth of separate Clinical Pharmacology departments. The TDM service in the country is broadly of two types: in large teaching hospitals where the service is available through departments of Clinical Pharmacology, and in the private sector, where drug estimations are done by clinical biochemistry departments with minimal interpretation. This article is based on literature review and our own experiences over a 10 year period in a department of Clinical Pharmacology. It focuses on the evolution of TDM, its problems such as lack of funding, special aspects such as the impact of ethnic differences, nutritional deficiencies, quality of medicines and availability of generic products; its utility as a research tool and its future.
\end{abstract}

Keywords: developing country, therapeutic drug monitoring

\section{Introduction}

Therapeutic Drug Monitoring (TDM) can be defined as the use of drug measurements in biological fluids as an aid to the management of patients receiving drug therapy for the alleviation or prevention of diseases. In the developed world, the evolution of TDM can be traced through three distinct phases - the 1960s which saw the development of the principles of TDM, the 1970s with automation of laboratory methods and the 1980s when there was widespread expansion of TDM [1]. In the 1990s the exuberance has given way to calculated restraint about the use of TDM as seen for example with antiepileptic drugs [2]. In an era where there are continuing pressures to provide services at the lowest possible cost, pharmacoeconomic considerations weigh down heavily upon TDM.

In all these respects, the developing countries are very different. TDM as a service (an adjunct to patient care) was introduced towards the mid and late 1980s and the last 10 years have seen it grow, along with the growth of separate Clinical Pharmacology departments. The service is being improved each year to include more and more drugs and the number of requests for TDM is increasing. Automation and the availability of technically simple methods have made this service available even in rural areas. Despite this, TDM in several centres remains within the confines of clinical biochemistry departments

Correspondence: Dr N. A. Kshirsagar, Seth Gordhandas Sunderdas Medical College and King Edward VII Memorial Hospital, Parel, Mumbai 400012 , India. Tel: 9 I 22 4I3 3767; Fax: 9122 4I4 3435; E-mail: dcpkem@vsnl.com that provide only the 'measuring' (assay only) and not the 'monitoring' (assay and clinical interpretation) service [3]. The ideal TDM team-a clinical pharmacologist, clinical pharmacist and analytical scientist exists in only a few places. In addition data on levels obtained are limited and physicians are not adequately trained to interpret and use the results optimally.

The present article is an overview of status of TDM in India and other developing countries; its evolution, problems, special aspects, and the promise it holds for developing countries in the future.

\section{TDM in our hospital and elsewhere in India}

Our TDM service began in a small way in 1988 with a single high performance liquid chromatograph (h.p.l.c.) and one research assistant in a tiny laboratory tucked away in a corner of an 1800 bed teaching public hospital. In 1992 we reported our experiences in the management of epilepsy in a developing country [4]. Over the last 10 years the laboratory has grown and is now part of a fully fledged department of Clinical Pharmacology with a total staff of 30, three h.p.l.c.s, and an automated immunoassay laboratory.

Our TDM service started with the monitoring of three anticonvulsants - phenytoin, phenobarbitone and carbamazepine as an adjunct to the epilepsy clinic run by the Neurology Department. Today the TDM outpatient clinic is run twice a week and has an annual attendance of 1500 new cases and 3000 old cases. The laboratory has three technicians, one pharmacist, and one medical officer who are involved in initial history taking, sample 
collection and analysis by h.p.l.c. The 'monitoring', not 'measuring', service is manned by three senior Clinical Pharmacologists who provide advice on dosage adjustment, nonresponsiveness, compliance, managing and identifying adverse reactions and using anticonvulsants in pregnancy.

In developing countries TDM services are broadly of two types: one is like ours in large teaching hospitals where the service is available through departments of Clinical Pharmacology, while the other is in the private sector, where the drug estimations are performed by the clinical biochemistry departments. The h.p.l.c. technique, which is used by teaching hospitals, is labour intensive, technically demanding and the turnaround time is high. However, as the consumables are available locally, the recurring cost is low. As the TDM service is provided by utilizing the same infrastructure as for other academic and commercially required studies (e.g. new drug pharmacokinetics, bioavailability); it can be offered at very low charge to the patient. For example we charge f0.5 per sample which includes simultaneous estimation of phenytoin, phenobarbitone and carbamazepine [5]. This is a cost effective proposition particularly for patients taking multiple anti convulsants.

Most clinical biochemistry departments use automated equipment and ready to use kits using the fluorescence polarization (FPIA) and enzyme mediated immunoassay (EMIT) techniques. The kits have to be imported and storage conditions properly ensured and the cost per drug test is usually $f^{3-4}$. The advantage here is short turnaround times and ease of use. However, these laboratories provide no clinical interpretation of data.

\section{Laboratory results -interpretation and recommendations}

Results reported from the clinical biochemistry laboratories give the values and the 'normal' or reference range. In services run by departments of Clinical Pharmacology, laboratory values, interpretation, remarks, and advice are also given. Some issues peculiar to developing countries (which may not now be so unusual in developed ones) need to be noted while interpreting the results.

\section{Special issues is developing countries}

(a) Alternative systems of medicine India is unique in having at least three systems of medicine coexisting with 'western' medicine (allopathy); ayurveda, homeopathy and unani. Some allopathic practitioners often coprescribe medicines from the alternative systems particularly for chronic disorders. Our own experience in the TDM clinic identified an interaction with 'shankhapushpi' an ayurvedic preparation pur- ported to be an anti epileptic and memory enhancer. A patient with a history of generalized tonic-clonic (GTC) seizures, well controlled and with plasma phenytoin levels within the therapeutic range, presented with sudden loss of seizure control. History revealed that he was taking 'shakhapushpi' and plasma analysis showed that his phenytoin level had dropped. Experimental studies showed that this drug had both pharmacokinetic and pharmacodynamic interactions with phenytoin [6].

Two other interesting patients who presented to the TDM clinic had GTC epilepsy and had switched over to 'ayurvedic' tablets and discontinued their anticonvulsant medication. The patients had both phenytoin and phenobarbitone detectable in their plasma and analysis of the tablets showed that they contained a combination of phenytoin and phenobarbitone [7]. Herbal medicines are being used by an increasing number of patients world wide, who may not necessarily advise their clinicians of the concomitant use. Interaction with conventional drugs have been documented for licorice, ginseng, tannic acids, plantain, uzara root, hawthorn and kyushin all of which may be prescribed by practitioners of the alternative systems [8].

(b) Tropical diseases and nutritional deficiencies Ill health is a serious problem impeding progress in most developing countries [9]. This includes diseases highly prevalent in these countries such as infections, diarrhoea, worm infestations, tuberculosis, neurocysticercosis and nutritional deficiencies, plus a higher proportion of patients with diabetes and AIDS. Patients often seek treatment late in their illness. Nutritional deficiencies are often subclinical and escape detection and they have been shown to affect drug pharmacokinetics [10].

Felder \& Stewart [11] have shown that the rural black population in South Africa often has albumin concentrations below the accepted reference range of $35-50 \mathrm{~g} \mathrm{l}^{-1}$. In a study which estimated free and total phenytoin and albumin levels in these patients, they were able to show that, because albumin levels are lower, corrected phenytoin concentrations using the Sheiner Tozer equation should be used and not the total phenytoin concentrations which can be misleading [11]. However, in our urban situation, over 100 consecutive patients screened were found to have normal albumin levels. Iron deficiency anaemia is common and may affect drug metabolism and absorption although we have not found it to affect phenytoin pharmacokinetics [12]. AIDS is a major problem in the developing world, with India estimated to have the largest number of cases of any 
country in the world. AIDS has been shown to reduce the absorption of antituberculous drugs and there are specific recommendations for monitoring antituberculin drug levels in these patients [13].

(c) Ethnic differences and extrapolation of the normal range The fact that interpopulation variations in drug pharmacokinetics can result in higher or lower plasma drug concentrations is well known. For example, the metabolism of phenytoin via para-hydroxylation is subject to wide interindividual variation. Mani has reported that the effective anticonvulsant dosage may be lower in Indians than in Europeans [14] while other authors have indicated that ethnic differences may have a significant influence on the plasma clearance of phenytoin.

Shelley studied possible ethnic differences in the pharmacokinetics of lithium carbonate in Caucasian and Afro-Caribbean volunteers under standardized conditions. There was a non statistically significant trend towards more rapid distribution and elimination, smaller area under the serum time-concentration curve and greater urinary excretion in the Caucasian group [15]. Lee studied the variability in plasma phenobarbitone concentration in Asian children in Singapore. This included Chinese, Malays and Indians and the mean phenobarbitone dosage required to produce a plasma level of $15 \mu \mathrm{g} \mathrm{ml}^{-1}$ was $5.2 \mathrm{mg} \mathrm{kg}^{-1} \mathrm{day}^{-1}$ and varied between the three groups although the differences were not statistically significant [16].

The standard therapeutic ranges for interpretation of TDM data are derived from population studies in the west. Nomograms used for dosage calculations for phenytoin have been made using pharmacokinetic data from developed countries but the same nomograms are used in developing countries. When we compared the expected phenytoin values (using the nomogram) with actual phenytoin values in our patients after dose adjustment; we found 10-15\% lower levels than calculated values. This could be accounted for by pharmacokinetic differences or different formulations with lower bioavailability [17].

(d) Quality control in drug assays For TDM programs, quality control is vitally important [3] and in developing countries there are hardly any procedures for laboratory accreditation or external quality control. In India, one centre in Southern India offers an external quality control program (for biochemical tests). For drug levels, however, there is none and most departments and laboratories such as ours use overseas quality control programs although this increases the cost of running the laboratory. In view of the mushrooming of private 'pathobiochem' laboratories which offer a range of pathology and biochemical investigations, the state Food and Drug Administrations are proposing laboratory inspections for standardizing and ensuring quality of results. There are no such proposals for drug assay laboratories.

(e) Quality of medicines and generic formulations World wide, there is increasing prescription of generic products which are actively promoted by health authorities for economic reasons. The prescription of generics by primary care physicians has risen in England from 35\% in 1985 to 55\% in 1995 [18]. Quality of products (drug content, bioavailability) is important especially for drugs with a narrow margin of safety which is just those drugs for which TDM is relevant.

In developing countries, there is a constant attempt to provide drugs to the majority of the population at low cost and bioavailability studies are done only at the time of obtaining marketing approval. Authors have already reported from Pakistan and Vietnam that quality of drugs used may be substandard and need additional quality control [19, 20]. Given that generic drugs are freely available in developing countries, quality assurance of manufacturing practice is essential [21]. The TDM service can be used to provide an important early indication of substandard drugs. For example, we have identified substandard products by observing low levels of phenytoin in patients otherwise known to be compliant and previously having levels in the therapeutic range.

\section{Drugs for which TDM may be indicated}

The drugs for which TDM is performed in developing countries are selected on the basis of total workload, cost effectiveness and cost benefit. Anticonvulsant drugs have been monitored most extensively.

\section{(a) TDM of anticonvulsants}

The most common indication for TDM of anticonvulsants has been nonresponse to a standard dose of drug. This allows differentiation between noncompliance, need for a higher dose and true drug resistance requiring change over to second drug. Other common indications include suspected drug toxicity, dose adjustment in pregnancy and drug interactions (especially common with antituberculous drugs). Correlating the patients' signs and symptoms with plasma concentrations has resulted in maximum benefit to the patient. Appropriate dosage 
adjustment and achieving therapeutic concentrations results in over $70 \%$ of epileptic patients getting good seizure control [22]. We noted that nearly $20 \%$ of our patients needed dose increments of only 25 or $50 \mathrm{mg}$ to achieve the desired therapeutic concentration. Studies on the use of anticonvulsants in children have been infrequent. Singh et al., in 20 children receiving either phenytoin alone or in combination with phenobarbitone, showed wide interindividual variation in the steady state levels of phenytoin [23]. Our own study in 108 children referred for TDM showed that at the first estimation, $3 / 23$ on phenytoin, $20 / 31$ on phenobarbitone and 15/25 on carbamazepine had plasma concentrations in the therapeutic range. 92\% children required dosage adjustment to achieve optimal concentrations highlighting the need for greater referral for TDM in this age group [24].

\section{(b) TDM of antituberculin drugs}

Tuberculosis is highly prevalent in developing countries and there is growing concern about drug resistance. The available data suggest that selected patients with tuberculosis and mycobacterium avium complex (MAC) fail to respond to therapy because they malabsorb their medication. In particular, patients with AIDS and known gastrointestinal diseases have been shown to have problems absorbing these drugs. TDM of anti mycobacterial drugs offers the clinician a chance to ensure that the patient achieves a serum concentration above the minimum inhibitory concentration [25]. The Madras tuberculosis centre in South India has developed a urine test to identify noncompliance with isoniazid therapy [26]. Recently, in one charitable dispensary, where generic rifampicin was being given, several nonresponders were detected. The bioavailability of the generic rifampicin was found to be comparable with the standard preparation (unpublished data). TDM along with in vitro resistance testing would have probably helped in this situation. However, whether low levels are due to poor compliance, poor formulation or poor absorption needs to be investigated further. TDM for anti mycobacterial drugs is still not widely available.

\section{(c) Serum lithium monitoring}

As with other drugs that require TDM, there is debate about the need for, and the frequency of routine serum lithium estimations. Levels of $0.5-0.8 \mathrm{mmol}^{-1}$ are recommended for long-term therapy of bipolar disorder. Concentrations lower than $0.5 \mathrm{mmol}^{-1}$ may be effective in some elderly patients while higher than $0.8 \mathrm{mmol}^{-1}$ may be needed in younger patients [27]. With the widespread availability of equipment to assay lithium, lithium TDM is being done more frequently.
Lithium monitoring in developing countries has focused on aspects such as compliance, patient education, ethnic differences, and predicting response to treatment. Shanming (1981) considered that one of the reasons for the restricted use of lithium in China may be the lack of facilities for monitoring serum lithium levels [28]. It has been emphasized that there is an urgent need to improve available laboratory facilities in mental health institutions, to encourage research in biological psychiatry and allow for cross cultural comparisons between the developed and the developing countries [29]. Lee [30], in a study of 50 Chinese patients attending the first lithium clinic in Hongkong showed that a mean serum lithium level of $0.63 \mathrm{mmol}^{-1}$ was seen. Laboratory monitoring however, was haphazard and polypharmacy abounded.

Authors have also attempted to extend the scope of lithium monitoring beyond serum. Verghese showed a high correlation between lithium levels in serum and saliva which was similar to patients from North America [31]. Khare felt that though there was a positive correlation between lithium concentrations in serum and saliva, there was a subgroup of patients who showed a better correlation than others [32].

\section{(d) TDM of cyclosporin}

The bioavailability of critical care drugs such as cyclosporin is extremely important and there may be ethnic differences in its pharmacokinetics. Raj et al. studied 30 consecutive adult patients who underwent renal transplantation with regular blood cyclosporin monitoring [33]. The patients were treated initially with $7 \mathrm{mg} \mathrm{kg}^{-1}$ and the dose titrated in response to blood levels. The cyclosporin dose was brought down to $1 \mathrm{mg} \mathrm{kg}^{-1}$. This study showed that Indian patients required a relatively lower dose than Caucasians to achieve optimum blood concentrations. The blood levels obtained were between 387 and $2120 \mathrm{ng} \mathrm{ml}^{-1}$ and there was no evidence of rejection or irreversible nephrotoxicity.

\section{Benefits of the TDM service}

The rewards of our TDM anticonvulsant service have come by way of patients who are seizure free, who are able to hold good jobs or epileptic men and women who have married and had children. Two years ago, we decided to see if this service benefited patients as much as we believed it did. A pharmacoeconomic analysis was done which calculated the direct and indirect costs of the service and the cost to the patient showed a significant benefit in those in whom therapeutic drug monitoring was carried out. Benefits included reduction in number of seizures, reduction in adverse drug reactions and better scholastic performance compared with those in whom 
TDM was not carried out. All other methods of management remained identical.

The TDM service, as well as directly benefitting the patients has led to important findings. Attempts at dose adjustments of phenytoin using TDM identified the need for $25 \mathrm{mg}$ tablets for better dosing. These tablets which were non existent when we started the service, are now marketed [34]. We also noted that in samples from neonates and infants, phenytoin concentrations were often below the therapeutic range and found that higher doses of phenytoin (10-15 $\mathrm{mg} \mathrm{kg}^{-1}$ ) than standard textbook recommendations are needed for this population [35].

Some drug interactions, which can be anticipated and avoided from knowledge of pharmacology, were noted to be frequently occurring, e.g. calcium tablets prescribed with phenytoin and proconvulsant antibacterials such as ciprofloxacin and mefloquine prescribed to epileptic patients.

The clinical pharmacologist's copious notes on the interpretation of TDM results can (and should) form excellent educational material. We were very pleased to note a medical student we had taught was working in the emergency room and referred a convulsing patient to the neurologist. He remarked that the patient was likely to be non compliant, and that a blood sample should be sent immediately to the Clinical Pharmacology department for TDM, and the dose adjusted bearing in mind the nonlinear kinetics of phenytoin!

TDM can also be used as a useful research tool. In the early 1990s, with the resurgence of malaria in the city, chloroquine resistant strains began emerging as a major problem. We started the estimation of plasma levels of chloroquine and its metabolite desethyl chloroquine and we were able to prove that the non responsiveness to chloroquine in these patients was due to parasite resistance and not to subtherapeutic levels. This was true both for drug resistant vivax and falciparum malaria. The Malaria Clinical Pharmacology outpatient department runs daily and monitors and treats all malaria cases. In addition, referrals for resistance testing also come from all over the city. We have also had patients with severe life threatening drug resistant malaria who have been treated successfully when TDM of chloroquine has been used as an adjunct to therapy $[36,37]$.

\section{The future}

Developing countries differ from developed ones in having weak health-care structures, inadequate financial resources, unreliable supply and quality of pharmaceuticals, lack of adequate drug legislation and policy and a high rate of inappropriate self-medication [38]. Priorities for health services are radically different from those in developed nations; for example TDM of anticonvulsants has a very low priority and may not be asked for even once during the entire treatment period in a patient with epilepsy.

Funding for drug monitoring services is hard to come by given the dedicated staff and equipment required. It is necessary to overcome the initial barrier; a questioning of the necessity and the utility of such a service. With our TDM service, which began in a very small way, we have been able to use the principles of clinical pharmacology as well as successfully educate physicians and medical students about them. Today our TDM service includes in addition to phenytoin, phenobarbitone and carbamazepine, lithium, cyclosporin, and valproic acid. Our department routinely carries out testing of generic formulations. Interestingly, the nephrologists of the hospital do not prescribe a generic cyclosporin unless its bioavailability has been tested and proven by us. At a time when cost considerations in the west have led to pooling of resources between clinical biochemistry and clinical pharmacology departments [39], in this country, the discipline of clinical pharmacology is still fairly new with only a few hospitals having fully-fledged departments. The ability of this discipline to be able to contribute in practically every area of medicine and add 'value' to the existing facilities remains its greatest asset. TDM particularly in a developing country forms an indispensable arm of this discipline.

\section{References}

1 Therapeutic drug monitoring and clinical biochemistry. eds Hallworth MJ, Capps NE, ACB Venture Publications, London, pp 1-28, 1993.

2 Eadie MJ. Therapeutic drug monitoring-anti epileptic drugs. Br J Clin Pharmacol 1998; 46: 185-193.

3 Gross AS. Best practice in therapeutic drug monitoring. Br J Clin Pharmacol 1998; 46: 95-99.

4 Kshirsagar NA, Shah PU. Management of epilepsy in developing countries. In Recent Advances in Epilepsy, eds Pedley TA, Meldrum BS. 5th edn. London: Churchill Livingstone. 1992: 159-176.

5 Joshi MV, Pohujani SM, Kshirsagar NA, Shah PU, Acharya VN. Simultaneous estimation of phenytoin, phenobarbitone, and carbamazepine. Ind J Pharmacol 1990; 22: 177-179

6 Dandekar UP, Chandra RS, Dalvi SS, et al. Analysis of a clinically important interaction between phenytoin and Shankhapushpi, an ayurvedic preparation. J Ethnopharmacol 1992; 35: 285-288.

7 Gogtay NJ, Rane CT, Dalvi SS, Shah PU, Kshirsagar NA. A story of ayurvedic tablets and misled epileptic patients. J Assoc Phys Ind 1999; 47: 1116.

8 Miller LG. Herbal medicinals: selected clinical considerations focusing on known or potential drug-herb interactions. Arch Int Med 1998; 158: 2200-2211.

9 Hamilton JR. Health research in the developing world: a 
gastroenterological view from Bangladesh. Can J Gastroenterol 1997; 11: 94-98.

10 Krishnaswamy K. Nutrition and drug metabolism. Ind J Med Res 1978; 68(Suppl): 109-120.

11 Felder C, Stewart MJ. Plasma total phenytoin: a possibly misleading test in developing countries. Ther Drug Monit 1999; 21: 155-160.

12 Joshi MV, Pohujani SM, Mehta BC, Acharya VN, Kshirsagar NA. Effect of iron deficiency anemia and its treatment on single dose phenytoin bioavailability. Eur J Clin Pharmacol 1993; 45: 387-388.

13 Havlir DV, Barnes PF. Tuberculosis in patients with human immunodeficiency virus infection. N Engl J Med 1999; 340: 367-373.

14 Mani KS. Collaborative epidemiological study on epilepsy in India. Final report of the Bangalore centre, Department of Neurology, National Institute of mental health and Neurosciences, Bangalore 1987.

15 Shelley RK. Are there ethnic differences in lithium pharmacokinetics and side effects? Int Clin Psychopharmacol 1987; 2: 337-342.

16 Lee HS. Variability of plasma phenobarbitone concentration in Asian children in Singapore. Ther Drug Monit 1984; 6: 182-188.

17 Joshi MV, Pohujani SM, Jain SK, Kshirsagar NA, Shah PU, Acharya VN. Phenytoin dose adjustments in Indian population. Neurol Ind 1990; 38: 339-342.

18 Johnston A, Holt DW. Generic substitution for cyclosporine: What should we be looking for in new formulations? Transplant Proc 1998; 30: 1652-1653.

19 Qureshi H, Ahmed W, Mehdi I. Locally produced drugs need quality control. J Pak Med Assoc 1998; 48: 226-227.

20 Cong LD, Yen PT, Nhu TV, Binh LN. Use and quality of anti malarial drugs in the private sector in Vietnam. Bull World Health Org 1998; 76: 51-58.

21 Anonymous. Generic medicines-can quality be assured? Drugs Ther Bull 1997; 35: 9-11.

22 Karande SC, Joshi MV, Kshirsagar NA, Shah PU. An analysis of epileptic patients non responsive to drugs. $J$ Assoc Phys Ind 1992; 40: 445-447.

23 Singh LM, Mehta S, Vohra RM, Nain CK. Monitoring of drug therapy in epileptic children. Int J Clin Pharmacol Ther Toxicol 1987; 25: 251-254.
24 Karande SC, Dalvi SS, Kshirsagar NA. Shortcomings in the pharmacotherapy of epileptic children in Bombay, India. J Trop Ped 1995; 41: 247-249.

25 Peloquin CA. Using therapeutic drug monitoring to dose the anti mycobacterial drugs. Clin Chest Med 1997; 18: $79-87$.

26 Sarma GR, Immaneul C, Kailasam S, Kannapiran M, Nair NG, Radhakrishna S. A modified method for the estimation of acetylisoniazid in urine. Ind J Med Res 1974; 62: 945-952.

27 Schou M. Forty years of lithium treatment. Arch Gen Psych 1997; 54: 9-13.

28 Shanming Y. Lithium therapy in China. Brief communication. Acta Psychiat Scand 1981; 64: 270-272.

29 Abidoun OA. The role of laboratory medicine in psychiatry. East Afr Med J 1991; 68: 389-399.

30 Lee S. The first lithium clinic in HongKong: a Chinese profile. Aust NZ J Psych 1992; 26: 450-453.

31 Verghese A, Indrani N, Kuruvilla K, Hill PG. Usefulness of saliva lithium estimation. Br J Psychiat 1977; 130: 148-150.

32 Khare CB, Sankaranarayanan A, Goel A, Khandelwal SK, Srinivasa Murthy R. Saliva lithium levels for monitoring lithium prophylaxis of manic depressive psychosis. Int J Clin Pharmacol Ther Toxicol 1983; 21: 451-453.

33 Raj DS, Somiah S, Mani K. Optimal cyclosporine therapyan Indian experience. J Assoc Phys Ind 1996; 44: 34-36.

34 Kshirsagar NA, Joshi MV, Shah PU, Dalvi SS. Need for $25 \mathrm{mg}$ tablets of phenytoin. J Assoc Phys Ind 1991; 39: 395-396.

35 Rane CT, Gogtay NJ, Kadam VS, Powar HS, Dalvi SS, Kshirsagar NA. Subtherapeutic levels of phenytoin with standard doses in infants: need to review dosage schedule. Br J Clin Pharmacol 1999; 48: 465-466.

36 Gogtay NJ, Garg MR, Bodhe PV, Tilve GH, Kshirsagar NA. Response of vivax malaria to chloroquine in mumbai. J Assoc Phys Ind 1998; 46: 843-845.

37 Garg MR, Gopinathan N, Bodhe PV, Kshirsagar NA. Vivax malaria resistant to chloroquine: case reports from Bombay. Trans Roy Soc Trop Med Hyg 1995; 89: 656-657.

38 Heaney D, Josemir WAS. Anti epileptic drugs in developing countries. Lancet 1998; 351: 1967.

39 Shenfield GM. Therapeutic drug monitoring beyond 2000. Br J Clin Pharmacol 1998; 46: 93-94. 